\title{
Binding energy of two-dimensional biexcitons
}

\author{
Singh, Jai; Birkedal, Dan; Vadim, Lyssenko; Hvam, Jørn Märcher
}

Published in:

Physical Review B

Link to article, DOI:

10.1103/PhysRevB.53.15909

Publication date:

1996

Document Version

Publisher's PDF, also known as Version of record

Link back to DTU Orbit

Citation (APA):

Singh, J., Birkedal, D., Vadim, L., \& Hvam, J. M. (1996). Binding energy of two-dimensional biexcitons. Physical Review B, 53(23), 15909-15913. https://doi.org/10.1103/PhysRevB.53.15909

\section{General rights}

Copyright and moral rights for the publications made accessible in the public portal are retained by the authors and/or other copyright owners and it is a condition of accessing publications that users recognise and abide by the legal requirements associated with these rights.

- Users may download and print one copy of any publication from the public portal for the purpose of private study or research.

- You may not further distribute the material or use it for any profit-making activity or commercial gain

- You may freely distribute the URL identifying the publication in the public portal

If you believe that this document breaches copyright please contact us providing details, and we will remove access to the work immediately and investigate your claim 


\title{
Binding energy of two-dimensional biexcitons
}

\author{
Jai Singh,* D. Birkedal, V. G. Lyssenko, ${ }^{\dagger}$ and J. M. Hvam \\ Mikroelektronik Centret, The Technical University of Denmark, DK2800 Lyngby, Denmark \\ (Received 16 October 1995; revised manuscript received 19 December 1995)
}

\begin{abstract}
Using a model structure for a two-dimensional (2D) biexciton confined in a quantum well, it is shown that the form of the Hamiltonian of the 2D biexciton reduces into that of an exciton. The binding energies and Bohr radii of a $2 \mathrm{D}$ biexciton in its various internal energy states are derived analytically using the fractional dimension approach. The ratio of the binding energy of a 2D biexciton to that of a $2 \mathrm{D}$ exciton is found to be 0.228 , which agrees very well with the recent experimental value. The results of our approach are compared with those of earlier theories. [S0163-1829(96)02623-9]
\end{abstract}

\section{INTRODUCTION}

With the advancement of techniques such as four-wave mixing (FWM) spectroscopy to study the transient nonlinear response of low-dimensional semiconductor structures grown by molecular-beam epitaxy, it has become rather common to observe the biexcitonic features ${ }^{1-16}$ in quantum well structures. Only the heavy hole $(\mathrm{HH})$ biexciton peaks are observed in the photoluminescence spectra of $\mathrm{GaAs}$ quantum wells, ${ }^{1,8,15,16}$ but recently also the light hole (LH) biexcitons have been observed by FWM. ${ }^{15,16}$ From these observations ${ }^{15,16}$ the binding energies of $\mathrm{HH}$ and $\mathrm{LH}$ biexcitons have been estimated for different well widths, and the ratio of the binding energy of $\mathrm{HH}$ biexcitons $\left(E b^{x x}\right)$ to that of $\mathrm{HH}$ excitons $\left(E b^{x}\right)$ has been calculated. It is thus found that $E b^{x x} / E b^{x} \approx 0.2$ and it is independent of the quantum well width. To our knowledge, no theoretical results on biexcitons published so far have predicted such a high value of $E b^{x x} / E b^{x}$ for quasi-two-dimensional (2D) excitons and biexcitons confined in GaAs quantum wells.

In order to bring the discrepancy between theory and experiment into perspective, it may be desirable here to review briefly some of the results that have been published so far on the binding energy of biexcitons. It was first Wheeler ${ }^{17}$ who in 1946 considered theoretically the possibility of the existence of complex particles consisting purely of electrons and positrons and suggested that, although one electron bound to one positron is stable, a complex such as two electrons bound to two positrons should not be stable in three dimensions (3D). On the contrary, then Hylleraas and Ore ${ }^{18}$ showed that a positronium molecule (two electrons bound to two positrons) in 3D should also be stable, and using a variational calculation found that $E b^{x x} / E b^{x}=0.017$ for such a complex in the positronium limit, where the masses of the negative and positive particles are equal, $m_{e}^{*}=m_{h}^{*}$. Lampert $^{19}$ has then pointed out that a biexciton, which he called an excitonic molecule, should exist in 3D nonmetallic solids, and using Hylleraas and Ore's theory, he estimated its binding energy to be in the same range. The first experimental evidence of the existence of a complex such as an exciton bound to a neutral donor was reported by Haynes ${ }^{20}$ in $3 \mathrm{D}$ silicon, who estimated the ratio of the binding energy of the complex to that of an exciton to be 0.1 , much higher than predicted by Hyleraas and Ore's ${ }^{18}$ theory. This value of the ratio is usually referred to as Haynes rule for $3 \mathrm{D}$ biexcitons. A few years later Haynes ${ }^{21}$ also observed the existence of excitonic molecules (biexcitons) in $3 \mathrm{D}$ silicon. As the value of $E b^{x x} / E b^{x}$ predicted from the Hylleraas and Ore theory was very small, Haynes assumed the same ratio of 0.1 for biexcitons as he had found earlier ${ }^{20}$ for the neutral donor complex, and taking the exciton binding energy in $\mathrm{Si}, E b^{x}=$ $8 \mathrm{meV}$, he estimated the binding energy of biexcitons in 3D silicon as, $E b^{x x}=0.8 \mathrm{meV}$. As Hylleraas and Ore's calculations were done for a positronium molecule $\left(m_{e}^{*}=m_{h}^{*}\right)$, some improvements in the theory were made by calculating the 3D biexciton binding energy ${ }^{22-24}$ as a function of $\sigma=$ $m_{e}^{*} / m_{h}^{*}$. Akimoto and Hanamura ${ }^{23}$ have thus found that $E b^{x x} / E b^{x}$ decreases monotonically with $\sigma$ in 3D solids, from 0.31 at $\sigma=0$ ( hydrogenic limit) to 0.027 at $\sigma=1$ (positronium limit), which was still significantly lower than the experimental value. ${ }^{20}$ Further improvements in the theory were made by Brinkman, Rice, and Bell, ${ }^{24}$ who used a sixparameter variational function to calculate the binding energy of a 3D biexciton numerically as a function of $\sigma$, and found that $E b^{x x} / E b^{x}=0.029$ for $\sigma=1$, in close agreement with Akimoto and Hanamura's result.

In 1982 Miller et al. ${ }^{1}$ observed the existence of 2D biexcitons in GaAs quantum wells, and determined $E b^{x x}=1$ meV for a quantum well of width $81 \AA$. The value decreased with increasing well width. They also calculated the binding energy of 2D biexcitons using a method similar to that of Brinkman, Rice, and Bell ${ }^{24}$ for $3 \mathrm{D}$, which was later published in detail by Kleinman. ${ }^{25}$ To our knowledge, this is the only theoretical work that has dealt specifically with the $2 \mathrm{D}$ biexcitons and calculated $E b^{x x} / E b^{x}$ as a function of both $\sigma$ and quantum well width. Kleinman's theory yields $E b^{x x} / E b^{x}=0.564$ for $\sigma=0$ (hydrogenic limit), 0.15 for $\sigma=0.68$ (GaAs), and 0.14 for $\sigma=1$ (positronium limit) at zero quantum well width. As the value of $E b^{x x} / E b^{x}$ decreases only slightly, although constantly from 0.15 to 0.11 when the well width increases from 0 to $170 \AA$, Kleinman has identified this ratio with the Haynes factor of 0.1 in $3 \mathrm{D}$. This theoretical result produced as high a value of $E b^{x x} / E b^{x}$ as predicted by Haynes for 3D biexcitons, therefore Kleinman concluded correctly that the binding energy of 2D biexcitons has to be larger than that of 3D biexcitons. Since then, several papers ${ }^{6,10-12}$ have reported even higher 
values of the binding energy of quasi-2D biexcitons. More recently, ${ }^{16}$ we have reported the results of a systematic experimental determination of the binding energy of biexcitons, which gives a value of $E b^{x x} / E b^{x} \approx 0.2$, nearly independent of the quantum well width for $\mathrm{HH}$ biexcitons in GaAs quantum wells. In Ref. 16 we have also reported the result of the present theory, which gives $E b^{x x} / E b^{x}=0.228$, in good agreement with our experimental result. However, our experimental results ${ }^{16}$ deviate from Kleinman's results at least in two aspects: (1) A value of $E b^{x x} / E b^{x} \approx 0.2$ has been observed and (2) $E b^{x x} / E b^{x}$ is found to be independent of the quantum well width. As Kleinman's approach is based on an extension of the 3D approach, we believe that the discrepancy may be attributed to the dimensionality of the approach.

As in our earlier report ${ }^{16}$ only the main result could be given; the aim of this paper is to present the details of the theory of calculating the binding energy of a $2 \mathrm{D}$ biexciton assuming a model structure and using the fractional dimension approach. ${ }^{26-28}$ To our understanding, the fractional dimension approach has not yet been applied for calculating the binding energy of biexcitons in quantum wells. Our model is particularly applicable to those cases where the exciton and biexciton diameters are larger than the quantum well width. The main advantage of our approach is that it is extremely simple, and produces analytical results for the binding energy and Bohr radius of biexcitons as functions of $\sigma$.

\section{MODEL OF 2D BIEXCITONS}

The general Hamiltonian for a biexciton, $\hat{H}_{x x}$, consisting of four charged particles, two electrons, and two holes, can be written as

$$
\hat{H}_{x x}=-\frac{\hbar^{2}}{2 m_{e}^{*}}\left(\nabla_{e 1}^{2}+\nabla_{e 2}^{2}\right)-\frac{\hbar^{2}}{2 m_{h}^{*}}\left(\nabla_{1 h}^{2}+\nabla_{2 h}^{2}\right)+V,
$$

where $m_{e}^{*}$ and $m_{h}^{*}$ are the effective masses of electrons and holes, respectively, and

$$
V=V_{e 1 e 2}-V_{e 1 h 1}-V_{e 1 h 2}-V_{e 2 h 1}-V_{e 2 h 2}+V_{h 1 h 2},
$$

where $V_{i j}$ denotes the Coulomb interaction between the charge particles $i$ and $j[i, j=e 1, e 2, h 1$, and $h 2$ denote the two electrons and two holes in the biexciton] given by

$$
V_{i j}=\frac{e^{2}}{\epsilon\left|\boldsymbol{r}_{i}-\boldsymbol{r}_{j}\right|}
$$

where $\epsilon$ is the dielectric constant of the material. $\nabla_{i}^{2}$ is the Laplacian with respect to the electron and hole coordinates, $\boldsymbol{r}_{i} ; i=e 1, e 2, h 1$, and $h 2$.

For quantum well widths smaller than the biexcitonic diameter, the biexciton is confined into a $2 \mathrm{D}$ space. For such a situation we propose a geometrical model of a 2D biexciton as shown in Fig. 1, where the charge carriers instantaneously form a square that can only move in its plane, which is the same as the plane of the quantum well. As all four particle charges are equal in magnitude such a symmetrical structure can be considered to be quite justified and realistic. For such a structure, the first four of the six relative coordinates, denoted by $\boldsymbol{t}_{i}, i=1, \ldots, 6$, can be represented by vectors parallel

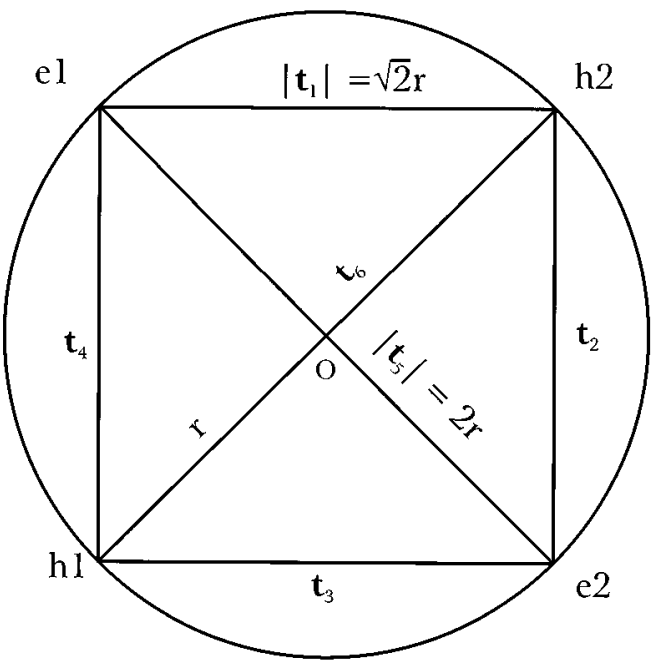

FIG. 1. A proposed model structure of a 2D biexciton confined in a quantum well of width narrower than the biexciton diameter.

to each of the sides and the last two parallel to the two diagonals of the square. It is obvious from Fig. 1 that the only way such a structure can be stable is when the repulsive Coulomb forces act along the diagonals of the square. Here, of course, we have assumed that the electron and hole lattice interactions are so small that they cannot cause any pairing of the like charges, which can take place in the case of strong lattice interaction as is well known. ${ }^{29}$

For our model structure of a 2D biexciton (Fig. 1), the relative coordinates can be written as

$$
\begin{gathered}
\boldsymbol{t}_{1}=-\left|\boldsymbol{r}_{e 1}-\boldsymbol{r}_{h 2}\right| \hat{\boldsymbol{i}}, \quad \boldsymbol{t}_{2}=\left|\boldsymbol{r}_{e 2}-\boldsymbol{r}_{h 2}\right| \hat{\boldsymbol{j}}, \\
\boldsymbol{t}_{3}=\left|\boldsymbol{r}_{e 2}-\boldsymbol{r}_{h 1}\right| \hat{\boldsymbol{i}}, \quad \boldsymbol{t}_{4}=-\left|\boldsymbol{r}_{e 1}-\boldsymbol{r}_{h 1}\right| \hat{\boldsymbol{j}}, \\
\boldsymbol{t}_{5}=\left|\boldsymbol{r}_{e 1}-\boldsymbol{r}_{e 2}\right|(\hat{\boldsymbol{i}}-\hat{\boldsymbol{j}}) / \sqrt{2}, \quad \boldsymbol{t}_{6}=\left|\boldsymbol{r}_{h 1}-\boldsymbol{r}_{h 2}\right|(\hat{\boldsymbol{i}}+\hat{\boldsymbol{j}}) / \sqrt{2},
\end{gathered}
$$

where $\hat{i}$ and $\hat{j}$ are two unit vectors parallel to the two adjacent sides of the square, and without the loss of any generality they can be considered parallel to the $x$ and $y$ directions in the $x y$ plane, which can also be assumed to be the plane of the quantum well. It is obvious that $\left|\boldsymbol{t}_{1}\right|=\left|\boldsymbol{t}_{2}\right|=\left|\boldsymbol{t}_{3}\right|=\left|\boldsymbol{t}_{4}\right|$ and $\left|\boldsymbol{t}_{5}\right|=\left|\boldsymbol{t}_{6}\right|$. Now by transforming the electron and hole coordinates into the relative coordinates and the center-ofmass coordinate, which is defined by

$$
\boldsymbol{R}=\frac{m_{e}^{*}\left(\boldsymbol{r}_{e 1}+\boldsymbol{r}_{e 2}\right)+m_{h}^{*}\left(\boldsymbol{r}_{h 1}+\boldsymbol{r}_{h 2}\right)}{M},
$$

where $M=2\left(m_{e}^{*}+m_{h}^{*}\right)$ is the total effective mass of the biexciton, the Hamiltonian (1) becomes ${ }^{30}$

$$
\hat{\mathbf{H}}_{x x}=-\frac{\hbar^{2}}{2 M} \nabla_{R}^{2}-\frac{\hbar^{2}}{2}\left(\frac{2}{\mu_{e h}} \nabla_{e h}^{2}+\frac{1}{\mu_{e e}} \nabla_{e e}^{2}+\frac{1}{\mu_{h h}} \nabla_{h h}^{2}\right)+V,
$$

where $1 / \mu_{e h}=1 / m_{e}^{*}+1 / m_{h}^{*}, \quad 1 / \mu_{e e}=2 / m_{e}^{*}, \quad$ and $1 / \mu_{h h}$ $=2 / m_{h}^{*} \cdot \nabla_{e h}^{2}, \nabla_{e e}^{2}$, and $\nabla_{h h}^{2}$ are the Laplacians with respect to the relative coordinates between electron and hole 
$\left(t_{i}, i=1, \ldots, 4\right)$, electron and electron $\left(t_{5}\right)$, and hole and hole $\left(t_{6}\right)$, respectively, and $\nabla_{R}^{2}$ is that with respect to the centerof-mass coordinate $\boldsymbol{R}$.

For calculating the internal energy eigenvalue of the biexciton, the first term of (6) can be ignored, being the kinetic energy operator of the center-of-mass motion. The rest of the Hamiltonian (6) can then be written in spherical coordinates by choosing the origin at the center of a circle in which the symmetric square of the biexciton structure can be inscribed (Fig. 1). This essentially means applying another coordinate transformation that can transform the relative coordinates as

$$
\left|t_{1}\right|=\left|t_{2}\right|=\left|t_{3}\right|=\left|t_{4}\right|=\sqrt{2} r
$$

and

$$
\left|\boldsymbol{t}_{5}\right|=\left|\boldsymbol{t}_{6}\right|=2 r
$$

where $r$ is the radius of the circle.

For calculating the binding energy of excitons confined in an $\alpha$-dimensional space, $1 \leqslant \alpha \leqslant 3$, recently a fractional dimensional approach has been applied. ${ }^{26-28}$ The advantage of the approach is that it produces an analytical expression for the exciton binding energy as a function of the dimensional parameter and hence a quantum well width that agrees reasonably well with the experimental result. We will follow this approach for calculating the binding energy of 2D biexcitons. Using Eq. (7) first we transform and then write the Hamiltonian (6) in spherical coordinates as a function of the fractional dimensional parameter ${ }^{26-28} \alpha$ as

$$
\hat{\mathbf{H}}_{x x}=-\left(\frac{\hbar^{2}}{2 \mu_{x x}}\right)\left[\frac{1}{r^{\alpha-1}} \frac{\partial}{\partial r}\left\{r^{\alpha-1} \frac{\partial}{\partial r}\right\}\right]+\frac{L^{2}}{2 \mu_{x x} r^{2}}-\frac{e^{2}}{\epsilon_{x x} r},
$$

where

$$
\mu_{x x}=\frac{2}{3} \mu_{e h}, \quad \epsilon_{x x}=\frac{\sqrt{2}}{4-\sqrt{2}} \epsilon .
$$

It is to be noted that for the present case $\alpha=2$, to conform with our proposed geometrical model of a $2 \mathrm{D}$ biexciton. $L^{2}$ is the square of the angular momentum operator of a biexciton, similar to that occurring in the 3D exciton or hydrogen atom Hamiltonian written in spherical coordinates. Thus by applying the above two transformations of coordinates and using our model structure, we have been able to reduce the Hamiltonian of a biexciton into that of a single exciton of a modified reduced mass, $\mu_{x x}$, and dielectric constant, $\epsilon_{x x}$, as given in Eq. (9).

\section{BIEXCITON BINDING ENERGY}

Here we will first outline very briefly the method of calculating the binding energy of a single exciton confined in low dimensions and then describe that for a biexciton. The binding energy of a single exciton in an $\alpha$-dimensional material can be derived by solving the following Schrödinger equation: ${ }^{26-28}$

$$
\hat{\mathbf{H}}_{x} \psi_{x, n}(r, \theta)=\left(E_{x, n}-E_{g}\right) \psi_{x, n}(r, \theta)
$$

where

$$
\hat{\mathbf{H}}_{x}=-\frac{\hbar^{2}}{2 \mu_{e h}} \frac{1}{r^{\alpha-1}} \frac{\partial}{\partial r}\left(r^{\alpha-1} \frac{\partial}{\partial r}\right)+\frac{L^{2}}{2 \mu_{e h} r^{2}}-\frac{e^{2}}{\epsilon r},
$$

and $E_{g}$ is the electronic energy gap of the material. $E_{x, n}$ and $\psi_{x, n}(r, \theta)$ are the energy eigenvalue and eigenfunction, respectively, of an exciton in its internal energy state of principal quantum number $n$. The solution of the Schrödinger equation (10) is obtained as $^{26-28}$

$$
E_{x, n}=E_{g}-\frac{E_{B}}{[n+(\alpha-3) / 2]^{2}} .
$$

One also gets the corresponding excitonic Bohr radius $a_{x, n}$ as

$$
a_{x, n}=\left[n+\frac{\alpha-3}{2}\right]^{2} a_{X}
$$

where

$$
E_{B}=\frac{\mu_{e h}}{\epsilon^{2} m_{e}} R_{H},
$$

and

$$
a_{X}=\frac{m_{e}}{\epsilon \mu_{e h}} a_{H} .
$$

$R_{H}$ and $a_{H}$ are the Rydberg constant and Bohr radius, respectively, and $m_{e}$ is the free electron mass. From (11) we get the binding energy of an exciton, $E b^{x}(n)$, in its $n$th energy state and confined in $\alpha$-dimensional space as

$$
E b^{x}(n)=\frac{E_{B}}{[n+(\alpha-3) / 2]^{2}} .
$$

According to (14), $E_{B}$ depends on $\sigma$, because $\mu_{e h}=m_{e}^{*} /(1+\sigma)$, and one can then write the binding energy (16) as a function of $\sigma$ as

$$
E b^{x}(n)=\frac{m_{e}^{*}}{m_{e} \epsilon^{2}(1+\sigma)} \frac{R_{H}}{[n+(\alpha-3) / 2]^{2}} .
$$

It is to be noted that usually $\alpha$ depends on the quantum well width, but in order to be consistent with our 2D model of biexcitons, we will stick to $\alpha=2$ for excitons as well. However, as the result (17) of the exciton binding energy is well known, ${ }^{26-28}$ we will not discuss it any further.

Now for calculating the binding energy of a $2 \mathrm{D}$ biexciton, we use the biexciton Hamiltonian (8) and solve the following Schrödinger equation:

$$
\hat{\mathbf{H}}_{x x} \psi_{x x, n}(r, \theta)=\left(E_{x x, n}-2 E_{g}\right) \psi_{x x, n}(r, \theta),
$$

where $E_{x x, n}$ and $\psi_{x x, n}$ are the energy eigenvalue and eigenfunction of a biexciton in its internal energy state $n$, respectively. As the Schrödinger equation (18) is analogous to Eq. (10), it can be solved analogously, and we get the biexciton energy eigenvalue as

$$
E_{x x, n}=2 E g-\frac{E_{B}^{\prime}}{[n+(\alpha-3) / 2]^{2}},
$$

and the corresponding biexciton Bohr radius $a_{x x, n}$ as 


$$
a_{x x, n}=\left(n+\frac{\alpha-3}{2}\right)^{2} a_{X}^{\prime},
$$

where $E_{B}^{\prime}$ and $a_{X}^{\prime}$ are obtained from (14) and (15) replacing $\mu_{e h}$ and $\epsilon$ by $\mu_{x x}$ and $\epsilon_{x x}$, respectively. The biexciton binding energy $E b^{x x}(n)$, defined as

$$
E b^{x x}(n)=2 E_{x, n}-E_{x x, n},
$$

is then obtained using (12) and (19) in (21) as

$$
E b^{x x}(n)=\left(\frac{E_{B}^{\prime}}{E_{B}}-2\right) E b^{x}(n) .
$$

Using Eq. (9) and the definition of $E_{B}^{\prime}$ in Eq. (22) we get

$$
E b^{x x}(n)=\left(\frac{(4-\sqrt{2})^{2}}{3}-2\right) E b^{x}(n),
$$

which gives

$$
\frac{E b^{x x}(n)}{E b^{x}(n)} \approx 0.228
$$

for $\alpha=2$ and it is independent of $\sigma$ and $n$. For simplification, denoting $E b^{x x}(n=1) / E b^{x}(n=1)$ by $E b^{x x} / E b^{x}$, we find that the value of $E b^{x x} / E b^{x}$ thus obtained in $2 \mathrm{D}$ is the highest that has so far been obtained theoretically, and it agrees very well with the experimental value ${ }^{15,16}$ of about 0.2 . The ratio (24) is slightly less than the double of the value (0.12) calculated by Kleinman ${ }^{25}$ for a GaAs $(\sigma=0.68)$ quantum well of width $81 \AA$ and more than double of the value known as Haynes rule ${ }^{20}$ for $3 \mathrm{D}$ semiconductors. Although our theoretical result is valid strictly in two dimensions, the fact that it agrees so well with the experimental value, which is nearly independent of the well width, does indeed suggest that the geometrical structure of a biexciton in quantum wells may not be very different from the one used in this calculation. This point will be addressed further in the next section.

Using (15) and (20) we get the ratio of the Bohr radius of biexcitons to that of excitons as

$$
\frac{a_{x x, n}}{a_{x, n}}=\frac{3(4-\sqrt{2})}{2 \sqrt{2}}=2.74 .
$$

Like the ratio of the binding energies, the ratio of Bohr radius of 2D biexcitons to that of excitons also does not depend on $\sigma$.

\section{DISCUSSION}

Using a simple but realistic model for the structural configuration of a 2D biexciton and applying two coordinate transfomations, it has been possible to transform the biexciton Hamiltonian into the form of an exciton Hamiltonian. The approach thus simplifies the biexciton problem extremely, and enables us to derive analytical expressions for the binding energy and Bohr radius of $2 \mathrm{D}$ biexcitons as a function of $\sigma$. The ratio of the binding energy of biexcitons to that of excitons, $E b^{x x} / E b^{x}$, and that of their Bohr radii, $a_{x x, n} / a_{x, n}$, has also been calculated. While both the binding energies and Bohr radii of 2D biexcitons and excitons depend individually on $\sigma$, their ratios (24) and (25) do not.
This is in contradiction with the results obtained for both 2D and 3D biexcitons, where the ratio $E b^{x x} / E b^{x}$ is known $^{23-25,31}$ to depend on $\sigma$, particularly in the limit $\sigma \rightarrow 0$, though for $\sigma>0$, the values of $E b^{x x} / E b^{x}$ obtained for 2D biexcitons in Ref. 25 decrease only slightly with $\sigma \rightarrow 1$. As the earlier calculation of $2 \mathrm{D}$ biexciton ${ }^{25}$ is carried out by extending the 3D theory, we believe that this slight difference may be attributed to the difference in the dimensionality of the two approaches. Moreover, as the dependence of $E b^{x x} / E_{b}^{x}$ on $\sigma$ is not studied experimentally, it is difficult to be any more conclusive about this point at present.

The binding energy of quasi-2D excitons in a GaAs quantum well of width $80 \AA$ is known ${ }^{16,26-28}$ to be about $10 \mathrm{meV}$, but it decreases with the quantum well width to its $3 \mathrm{D}$ value at large well widths. ${ }^{32}$ Using this value in Eq. (24) we find that the $2 \mathrm{D}$ biexciton binding energy can be about $2.3 \mathrm{meV}$, which agrees very well with the observed value of more than $2 \mathrm{meV} \cdot{ }^{15,16}$ No theoretical results, to our knowledge, have reported as high a value of the $2 \mathrm{D}$ biexciton binding energy as obtained here.

However, it may be argued that the present theoretical result being valid only for the exact $2 \mathrm{D}$ case cannot be applied to quantum wells for which usually $\alpha \geqslant 2$. This point can be addressed as follows: Experimentally, we know that the ratio $E b^{x x} / E b^{x}=0.1$ from the Haynes rule for the 3D case and about 0.2 for the quantum wells, which does not depend on the well width ${ }^{16}$ as long as the wells are narrower than the biexciton diameter. As one can see that our calculated result of $E b^{x x} / E b^{x}$ is slightly higher than the experimental value in quantum wells, we believe that the discrepancy is due to the difference in the dimensionality of the exact 2D situation and that of quantum wells. In this view the result of our calculation can be regarded as the upper limit for the ratio $E b^{x x} / E b^{x}$ in quantum wells, as indeed is the case, because the experimental value is only slightly lower than the exact $2 \mathrm{D}$ value but it is twice the $3 \mathrm{D}$ value. This also implies that our geometrical 2D biexciton model (Fig. 1) may be applied for quantum wells as well, because the 2D symmetrical structure may be possible not only at zero well width, but also at narrow well widths. The crucial point is the applicability of the two coordinate transformations [Eqs. (4) and (7)] used to reduce the 2D biexciton Hamiltonian into the form of an exciton Hamiltonian. We believe that these transformations will be applicable in quantum wells provided the well width is smaller than the biexcitonic diameter, which will not allow the biexciton to have the three-dimensional degrees of freedom. As the biexciton has a quantized energy state its configurational structure has to be quantized, which is a well-known result of the quantum mechanics. Using the same argument, it may be suggested that our geometrical 2D model may be applied to the real quantum wires as well for which usually $1<\alpha<2$. Thus within the boundary condition that the well width is less than the biexcitonic diameter, it may be implied that the theoretical value of $E b^{x x} / E b^{x}=0.228$ (24) is independent of the quantum well width, because it does not depend on $\alpha$, which is the only well-width-dependent parameter occurring in the binding energy. This is supported by the experimental result $^{16}$ that the ratio is independent of the well width and its value coincides remarkably well with the calculated $2 \mathrm{D}$ value. 
It is well known that the effect of the confinement increases the binding energy of an exciton. It is also known that in finite quantum wells, when the well width becomes quite narrow the exciton binding energy starts decreasing, because the exciton wave functions spread to the barrier regions and the $3 \mathrm{D}$ character $^{27}$ is restored. As the problem of biexcitons has been reduced into an exciton problem in our approach and a constant value of $E b^{x x} / E b^{x}$ has thus been obtained, the bixciton binding energy can be expected to behave in the same way as the exciton binding energy.

It may be recalled here that in the theory of excitons, when developed from a 3D multiparticle system of a condensed matter, the electronic Hamiltonian reduces to a twoparticle Hamiltonian given in (11) only after several approximations, in particular after neglecting the exchange interaction between the electron and hole. ${ }^{29}$ Likewise, for a biexciton we have started with a four-particle system and also neglected exchange interactions. Although other theories $^{23-25}$ of biexcitons have also considered the same potential as we have in (2), the contribution of the exchange

*Permanent address: Faculty of Science, Northern Territory University, Darwin, NT 0909, Australia.

†Permanent address: Institute of Microelectronics Technology, and Superpure Materials, Chernogolovka, Moscow District 142432, Russia.

${ }^{1}$ R.C. Miller, D.A. Kleinman, A.C. Gossard, and O. Munteanu, Phys. Rev. B 25, 6545 (1982).

${ }^{2}$ S. Charbonneau, T. Steiner, M.L.W. Thewalt, E.S. Koteles, J.Y. Chi, and B. Elman, Phys. Rev. B 38, 3583 (1988).

${ }^{3}$ L. Banyai, I. Galbraith, and H. Haug, Phys. Rev. B 38, 3931 (1988)

${ }^{4}$ R. Cingolani, Y. Chen, and K. Ploog, Phys. Rev. B 38, 13478 (1988).

${ }^{5}$ R. Cingolani, K. Ploog, G. Peter, R. Hahn, E.O. Göbel, C. Moro, and A. Cingolani, Phys. Rev. B 41, 3272 (1990).

${ }^{6}$ D. Oberhauser, K.-H. Pantke, W. Langbein, V.G. Lyssenko, H. Kalt, J.M. Hvam, G. Weiman, and C. Klingshirn, Phys. Status Solidi B 173, 53 (1992).

${ }^{7}$ D.J. Lovering, R.T. Phillips, G.J. Denton, and G.W. Smith, Phys. Rev. Lett. 68, 1880 (1992).

${ }^{8}$ R.T. Phillips, D.J. Lovering, G.J. Denton, and G.W. Smith, Phys. Rev. B 45, 4309 (1992).

${ }^{9}$ K.-H. Pantke, D. Oberhauser, V.G. Lyssenko, and J.M. Hvam, Phys. Rev. B 47, 2413 (1993).

${ }^{10}$ J.C. Kim, D.K. Wake, and J.P. Wolfe, Phys. Rev. B 50, 15099 (1994).

${ }^{11}$ S. Bar-Ad and I. Bar-Joseph, Phys. Rev. Lett. 68, 349 (1992); S. Bar-Ad, I. Bar-Joseph, G. Finkelstein, and Y. Levinson, Phys. Rev. B 50, 18375 (1994).

${ }^{12}$ G.O. Smith, E.J. Mayer, J. Kuhl, and K. Ploog, Solid State Commun. 92, 325 (1994); E.J. Mayer, G.O. Smith, V. Heuckeroth, J. Kuhl, K. Bott, A. Schulze, T. Meier, D. Bennhardt, S. W. Koch, P. Thomas, R. Hey, and K. Ploog, Phys. Rev. B 50, 14730 (1994)

${ }^{13}$ E.J. Mayer, G.O. Smith, V. Heuckeroth, J. Kuhl, K. Bott, A. Schulze, T. Meier, S.W. Koch, P. Thomas, R. Hey, and K. Ploog, Phys. Rev. B 51, 10909 (1995). interaction and other electronic correlations may be expected to reduce the value of $E b^{x x} / E b^{x}$. On the other hand, one may also expect some experimetal uncertainties to be involved.

The observed enhanced binding energy of biexcitons in GaAs quantum wells has also recently ${ }^{33}$ been interpreted on the basis of a polariton effect, which modifies the biexciton dispersion relations at small momenta and increases the biexciton binding energy. It has therefore been argued that the increased binding energy is due to the formation of bipolaritons instead of biexcitons in GaAs quantum wells. As the polariton effects go beyond the scope of the model calculations presented here, more experiments are needed to resolve this issue.

It is to be noted that the Bohr radius of biexcitons is more than two times larger than that of excitons. The Bohr radius of a biexciton may be regarded to be that of a circle around which all four charges move such that their instantaneous positions will be at the vertices of a square inscribed in the circle, as shown in Fig. 1.

${ }^{14}$ F. Keller, M. Lowisch, J. Puls, and F. Henneberger, Phys. Rev. Lett. 75, 2420 (1995).

${ }^{15}$ J. Erland, D. Birkedal, V.G. Lyssenko, and J.M. Hvam, in Radiative Processes and Dephasing in Semiconductors, edited by J. Kuhl and D. Citrin, Special issue of J. Opt. Soc. Am. B (to be published).

${ }^{16}$ D. Birkedal, J. Singh, V.G. Lyssenko, J. Erland, and J.M. Hvam, Phys. Rev. Lett. 76, 672 (1996).

${ }^{17}$ J.A. Wheeler, Ann. N.Y. Acad. Sci. 48, 221 (1946).

${ }^{18}$ E.A. Hylleraas and A. Ore, Phys. Rev. 71, 493 (1947).

${ }^{19}$ M.A. Lampert, Phys. Rev. Lett. 1, 450 (1958).

${ }^{20}$ J.R. Haynes, Phys. Rev. Lett. 4, 361 (1960).

${ }^{21}$ J.R. Haynes, Phys. Rev. Lett. 17, 860 (1966).

${ }^{22}$ R.R. Sharma, Phys. Rev. 170, 770 (1968); 171, 36 (1968).

${ }^{23}$ Q. Akimoto and E. Hanamura, Solid State Commun. 10, 253 (1972); J. Phys. Soc. Jpn. 33, 1537 (1973).

${ }^{24}$ W.F. Brinkman, T.M. Rice, and B. Bell, Phys. Rev. B 8, 1570 (1973).

${ }^{25}$ D.A. Kleinman, Phys. Rev. B 28, 871 (1983).

${ }^{26}$ X.F. He, Phys. Rev. B 43, 2063 (1991).

${ }^{27}$ H. Mathieu, P. Lefebvre, and P. Christol, Phys. Rev. B 46, 4092 (1992).

${ }^{28}$ P. Lefebvre, P. Christol, and H. Mathieu, Phys. Rev. B 48, 17308 (1993).

${ }^{29}$ J. Singh, Excitation Energy Transfer Processes in Condensed Matter-Theory and Applications (Plenum, New York, 1994), pp. 203-256.

${ }^{30}$ One can follow the usual procedure of transforming the partial differential operators. For example, consider the partial derivative with respect to the $x$ coordinate of electron, $e 1$. To transform into relative coordinates one can expand it as

$$
\frac{\partial}{\partial x_{e 1}}=\sum_{i} \frac{\partial x_{t_{i}}}{\partial x_{e 1}} \frac{\partial}{\partial x_{t_{i}}} ; \quad i=1, \ldots, 6 .
$$

${ }^{31}$ J. Adamowski, S. Bednarek, and M. Suffczynski, Solid State Commun. 9, 2037 (1971).

${ }^{32}$ A. Thilagam and J. Singh, J. Lumin. 55, 11 (1993).

${ }^{33}$ A.L. Ivanov and H. Haug, Phys. Rev. Lett. 74, 438 (1995). 